XAVIER BARDina (Barcelona)

CARles Rovira (Barcelona)

SAMY TINDEL (Paris)

\title{
ASYMPTOTIC EVALUATION OF THE POISSON MEASURES FOR TUBES AROUND JUMP CURVES
}

Abstract. We find the asymptotic behavior of $P(\|X-\phi\| \leq \varepsilon)$ when $X$ is the solution of a linear stochastic differential equation driven by a Poisson process and $\phi$ the solution of a linear differential equation driven by a pure jump function.

1. Introduction. This paper deals with the asymptotic evaluation of the Poisson measure for tubes around jump curves.

This problem has been widely studied for the Wiener measure: let $\left\{W_{t}\right.$; $t \geq 0\}$ be a Wiener process in $\mathbb{R}^{d}$ and $\phi \in L^{2}\left([0,1], \mathbb{R}^{d}\right)$. It is known that

$$
P\left(\|W-\phi\|_{\infty} \leq \varepsilon\right) \sim \frac{4}{\pi} \exp \left(-\frac{\pi^{2}}{8 \varepsilon^{2}}-\frac{|\phi|^{2}}{2}\right) .
$$

A related problem is the computation of Onsager-Machlup functionals: given a process $X$, we consider a norm $\|\cdot\|$ and two smooth curves $\phi$ and $\psi$. If

$$
\lim _{\varepsilon \downarrow 0} \frac{P(\|X-\phi\| \leq \varepsilon)}{P(\|X-\psi\| \leq \varepsilon)}
$$

2000 Mathematics Subject Classification: 60G17, 60H10.

Key words and phrases: Onsager-Machlup functionals, Poisson process.

Research of X. Bardina partially supported by grants CICYT No. BFM2000-0607, BFM2000-0009, HF1998-0096.

Research of C. Rovira partially supported by grants CICYT No. BFM2000-0607 and HF1998-0096.

Research of S. Tindel partially supported by grant CIES No. 99071. 
exists and can be expressed as

$$
\exp \left[\int_{0}^{T} L(\dot{\phi}(s), \phi(s)) d s-\int_{0}^{T} L(\dot{\psi}(s), \psi(s)) d s\right]
$$

for a given function $L(\dot{x}, x)$, then the above expression is called the OnsagerMachlup functional, and can be interpreted as a likelihood ratio for the law of the process $X$. Most of the studies on Onsager-Machlup's functional concern the case of a diffusion process $X$ which is the solution of the stochastic differential equation

$$
d X(t)=b(X(t)) d t+d W(t), \quad X(0)=x_{0}, \quad X(t) \in \mathbb{R}^{d},
$$

where $x_{0} \in \mathbb{R}^{d}$ and the coefficient $b$ has some regularity. Then, for a large class of norms on the Wiener space, and for functions in the CameronMartin space, it can be shown that the Onsager-Machlup function exists and is given by

$$
L(\dot{\phi}, \phi)=-\frac{1}{2} \sum_{i=1}^{d}\left|\dot{\phi}_{i}-b_{i}(\phi)\right|^{2}-\frac{1}{2} \sum_{i=1}^{d} \frac{\partial b_{i}}{\partial x_{i}}(\phi) .
$$

We refer to Ikeda and Watanabe (1981) and Shepp and Zeitouni (1992) for some basic results in that direction, and to Lyons and Zeitouni (1999) and Capitaine (2000) for theorems concerning the consistency of the OnsagerMachlup functional with respect to the norm considered on the Wiener space. Note also that the case of SDEs in infinite dimensions driven by a Gaussian noise have been considered in Mayer-Wolf and Zeitouni (1993), and Bardina, Rovira and Tindel (2000, 2001).

A natural (but to our knowledge unadressed in the literature) problem is to find if this kind of result still holds on the Poisson space. That is, if $N$ is a standard Poisson process on $[0,1], X$ the solution to a stochastic differential equation driven by $N$, and $\phi:[0,1] \rightarrow \mathbb{R}$ a deterministic function, we would like to evaluate $P\{\|X-\phi\| \leq \varepsilon\}$ for various norms. For the sake of computations, we deal with two simple cases: the case $X=N$, and the case when $X$ is the solution to a linear equation of the form

$$
X_{t}=\int_{0}^{t} X_{s} d s+N_{t}, \quad t \in[0,1] .
$$

In those two examples, some fundamental differences with respect to the Gaussian case can already be observed:

1. The Girsanov transform, which is an essential tool in the computation of the Onsager-Machlup functional on the Wiener space, is of little help in our case, since it transforms the Poisson process to a general semi-martingale that cannot be handled easily. 
2. It seems natural to deal with jump functions, which are the closest to the a.s. paths of the Poisson process. For this reason, we will evaluate the probability of some tubes around functions of the type

$$
h_{t}=\sum_{i=1}^{k} I_{\left[S_{i}, 1\right)}(t) \text {, }
$$

where $k \geq 0$ and $0<S_{1}<\ldots<S_{k}<1$ are the jump points of $h$ in the case $X=N$, and of the type

$$
\phi_{t}^{h}=\int_{0}^{t} \phi_{s}^{h} d s+h_{t}
$$

in case $X$ is the solution to the corresponding equation driven by the Poisson process.

3. From our results (see Theorems 3.1 and 4.1), it seems that the asymptotic evaluation of $P(\|X-\phi\| \leq \varepsilon)$ only depends on the number of jumps of $\phi$. This confirms the impression that $N$ is a "uniformly" distributed process when conditioned on its number of jumps.

Note also that our result in the SDE case is obtained by a linearization procedure. We hope to extend this method to a more general type of equations in a forthcoming paper.

Our article is organized as follows: in the next section, we will recall some very basic facts about the standard Poisson process $N$. Then we will compute the asymptotic evaluation for $N$ in Section 3, and for the linear SDE case in Section 4.

2. Preliminaries. Let $(\Omega, \mathcal{F}, P)$ be a complete probability space,

Definition 2.1. A Poisson process is a càdlàg process $N=\left\{N_{s} ; s \in\right.$ $[0,1]\}$ such that:

- $N_{0}=0$.

- Given $0 \leq t_{1}<\ldots<t_{n} \leq 1$ the increments

$$
N_{t_{n}}-N_{t_{n-1}}, N_{t_{n-1}}-N_{t_{n-2}}, \ldots, N_{t_{2}}-N_{t_{1}}, N_{t_{1}}
$$

are independent.

- Given $s<t$, the increment $N_{t}-N_{s}$ has a Poisson law of parameter $t-s$.

Given a Poisson process, we will denote by $T_{1}, T_{2}, \ldots$ the successive jump points of the Poisson process.

In this situation the following result is well known (see for instance Bhattacharya and Waymire, 1990, Proposition 4.5.6): 
Proposition 2.2. The conditional distribution of $\left(T_{1}, \ldots, T_{k}\right)$ given $\left\{N_{t}=k\right\}$ is the same as that of $k$ increasingly ordered independent random variables each having the uniform distribution on $(0, t]$. That is, the conditional density of the vector $\left(T_{1}, \ldots, T_{k}\right)$ given $\left\{N_{t}=k\right\}$ is

$$
f_{\left(T_{1}, \ldots, T_{k}\right)}\left(t_{1}, \ldots, t_{k}\right)=k ! I_{\left\{0<t_{1}<\ldots<t_{k}<t\right\}}\left(t_{1}, \ldots, t_{k}\right) .
$$

In this paper, given a standard Poisson process $\left\{N_{s} ; s \in[0,1]\right\}$ we will consider a diffusion process of the form

$$
X_{t}=\int_{0}^{t} X_{s} d s+N_{t}, \quad t \in[0,1] .
$$

It is easy to check that the solution of this equation can be written in terms of the jump points of the Poisson process as

$$
X(t)=\sum_{i=1}^{\infty} e^{t-T_{i}} I_{\left[T_{i}, 1\right)}\left(t_{i}\right) .
$$

3. The case of a Poisson process. When we consider a standard Poisson process, we have the following result:

Theorem 3.1. Let $\left\{N_{s} ; s \in[0,1]\right\}$ be a standard Poisson process and $h$ a real-valued jump function defined on $[0,1]$, with expression

$$
h_{t}=\sum_{i=1}^{k} I_{\left[S_{i}, 1\right)}(t),
$$

where $0<S_{1}<\ldots<S_{k}<1$ are the jump points. Then, for $\varepsilon>0$ small enough,

$$
\begin{aligned}
& P\left\{\|N-h\|_{1} \leq \varepsilon\right\}=2^{k} e^{-1}\left(e^{\varepsilon}-\sum_{j=0}^{k-1} \frac{\varepsilon^{j}}{j !}\right)=2^{k} e^{-1} \frac{\varepsilon^{k}}{k !}+O\left(\varepsilon^{k+1}\right), \\
& P\left\{\|N-h\|_{2} \leq \varepsilon\right\}=2^{k} e^{-1} \frac{\varepsilon^{2 k}}{k !}+O\left(\varepsilon^{2 k+2}\right) .
\end{aligned}
$$

REMARK 3.2. Note that the probability depends only on the number of jumps of the function $h$.

Proof. We will only develop the $L^{2}$ case. The proof for the $L^{1}$ norm can be done using the same arguments.

Since $0<S_{1}<\ldots<S_{k}<1$, there exists $\varepsilon_{0}$ such that $S_{i+1}-S_{i}>\varepsilon_{0}^{2}$ for all $i \in\{1, \ldots, k-1\}, S_{1}>\varepsilon_{0}^{2}$ and $1-S_{k}>\varepsilon_{0}^{2}$. Along the proof we will consider $\varepsilon<\varepsilon_{0}$. 
We have

$$
P\left\{\|N-h\|_{2} \leq \varepsilon\right\}=\sum_{j=0}^{\infty} P\left\{\|N-h\|_{2} \leq \varepsilon \mid N_{1}=j\right\} \frac{e^{-1}}{j !} .
$$

In order to compute the probabilities involved in the sum we will consider three cases:

- If $j<k$, then $P\left\{\|N-h\|_{2} \leq \varepsilon \mid N_{1}=j\right\}=0$. Indeed, in this case,

$$
\|N-h\|_{2}^{2} \geq \int_{S_{k}}^{1}\left(N_{t}-h_{t}\right)^{2} d t \geq \int_{S_{k}}^{1} d t=1-S_{k}>\varepsilon^{2} .
$$

- If $j=k$, then $P\left\{\|N-h\|_{2} \leq \varepsilon \mid N_{1}=k\right\}=\left(2 \varepsilon^{2}\right)^{k}$. We will prove this by induction on $k$. Notice that if $\left|T_{i}(\omega)-S_{i}\right|>\varepsilon^{2}$ for some $i \in\{1, \ldots, k\}$, then it is easy to check that $\|N(\omega)-h\|_{2}^{2}>\varepsilon^{2}$. Otherwise, if $\left|T_{i}(\omega)-S_{i}\right|<\varepsilon^{2}$ for all $i \in\{1, \ldots, k\}$, then

$$
\|N(\omega)-h\|_{2}^{2}=\sum_{i=1}^{k}\left|T_{i}-S_{i}\right| .
$$

So

$$
P\left\{\|N-h\|_{2} \leq \varepsilon \mid N_{1}=k\right\}=P\left\{\sum_{i=1}^{k}\left|T_{i}-S_{i}\right| \leq \varepsilon^{2} \mid N_{1}=k\right\} .
$$

For $k=1$, using Proposition 2.2 we have

$$
\begin{aligned}
P\left\{\left|T_{1}-S_{1}\right| \leq \varepsilon^{2} \mid N_{1}=1\right\} & =P\left\{S_{1}-\varepsilon^{2} \leq T_{1} \leq S_{1}+\varepsilon^{2} \mid N_{1}=1\right\} \\
& =\int_{S_{1}-\varepsilon^{2}}^{S_{1}+\varepsilon^{2}} d t=2 \varepsilon^{2} .
\end{aligned}
$$

Assume now that if $k=n$, then

$$
P\left\{\sum_{i=1}^{n}\left|T_{i}-S_{i}\right| \leq \varepsilon^{2} \mid N_{1}=n\right\}=\left(2 \varepsilon^{2}\right)^{n},
$$

and consider $k=n+1$. By Proposition 2.2, if $\varepsilon$ is small enough,

$$
\begin{aligned}
P\left\{\sum_{i=1}^{n+1}\left|T_{i}-S_{i}\right| \leq \varepsilon^{2} \mid\right. & \left.N_{1}=n+1\right\} \\
= & \int_{\left\{t_{1}<\ldots<t_{n+1}: \sum_{i=1}^{n+1}\left|t_{i}-S_{i}\right| \leq \varepsilon^{2}\right\}}(n+1) ! d t_{1} \ldots d t_{n+1} \\
& =(n+1) \int_{S_{n+1}-\varepsilon^{2}}^{S_{n+1}+\varepsilon^{2}}\left(n ! \int_{A_{\varepsilon}} d t_{1} \ldots d t_{n}\right) d t_{n+1},
\end{aligned}
$$


where

$$
A_{\varepsilon}=\left\{t_{1}<\ldots<t_{n} ; \sum_{i=1}^{n}\left|t_{i}-S_{i}\right| \leq \varepsilon^{2}-\left|t_{n+1}-S_{n+1}\right|\right\} .
$$

But, by the induction hypothesis the last expression is equal to

$$
\begin{aligned}
(n+1) 2^{n} \int_{S_{n+1}-\varepsilon^{2}}^{S_{n+1}+\varepsilon^{2}}\left(\varepsilon^{2}-\left|t_{n+1}-S_{n+1}\right|\right)^{n} d t_{n+1} & \\
= & (n+1) 2^{n} 2 \int_{0}^{\varepsilon^{2}}\left(\varepsilon^{2}-u\right)^{n} d u=2^{n+1} \varepsilon^{2(n+1)},
\end{aligned}
$$

which is the desired conclusion.

- Finally, if $j>k$ then $P\left\{\|N-h\|_{2} \leq \varepsilon \mid N_{1}=j\right\} \leq 2^{k} \varepsilon^{2 j}$.

Notice that if $\left|T_{i}(\omega)-S_{i}\right|>\varepsilon^{2}$ for some $i \in\{1, \ldots, k\}$, or if $1-T_{i}(\omega)>\varepsilon^{2}$ for some $i \in\{k+1, \ldots, j\}$, then $\|N(\omega)-h\|_{2}^{2}>\varepsilon^{2}$. Otherwise,

$$
\|N(\omega)-h\|_{2}^{2}=\sum_{i=1}^{k}\left|T_{i}(\omega)-S_{i}\right|+\sum_{i=k+1}^{j}\left(T_{i+1}(\omega)-T_{i}(\omega)\right)(i-k)^{2},
$$

where in order to simplify the notation we assume $T_{j+1} \equiv 1$.

But

$$
\begin{aligned}
\sum_{i=k+1}^{j}\left(T_{i+1}-T_{i}\right)(i-k)^{2} & =\sum_{i=k+1}^{j}\left[\left(1-T_{i}\right)-\left(1-T_{i+1}\right)\right](i-k)^{2} \\
& =\sum_{i=k+1}^{j}\left(1-T_{i}\right)(2(i-k)-1) \geq \sum_{i=k+1}^{j}\left(1-T_{i}\right) .
\end{aligned}
$$

So, in this case, for $\varepsilon$ small enough,

$$
\begin{aligned}
P\{\| N & \left.-h \|_{2} \leq \varepsilon \mid N_{1}=j\right\} \\
& =P\left\{\sum_{i=1}^{k}\left|T_{i}-S_{i}\right|+\sum_{i=k+1}^{j}\left(1-T_{i}\right)(2(i-k)-1)<\varepsilon^{2} \mid N_{1}=j\right\} \\
& \leq P\left\{\sum_{i=1}^{k}\left|T_{i}-S_{i}\right|+\sum_{i=k+1}^{j}\left(1-T_{i}\right) \leq \varepsilon^{2} \mid N_{1}=j\right\} .
\end{aligned}
$$

Using the result for the case $j=k$, and setting

$$
\psi\left(t_{k+1}, \ldots, t_{j}\right)=2^{k}\left(\varepsilon^{2}-\sum_{i=k+1}^{j}\left(1-t_{i}\right)\right)^{k},
$$


we find that the last expression is equal to

$$
\frac{j !}{k !} \int_{1-\varepsilon^{2}}^{1} \int_{1-\left(\varepsilon^{2}-\left(1-t_{j}\right)\right)}^{t_{j}} \ldots \int_{1-\left(\varepsilon^{2}-\sum_{i=k+2}^{j}\left(1-t_{i}\right)\right)}^{t_{k+2}} \psi\left(t_{k+1}, \ldots, t_{j}\right) d t_{k+1} \ldots d t_{j}=2^{k} \varepsilon^{2 j} .
$$

Thus,

$$
\begin{aligned}
P\left\{\|N-h\|_{2} \leq \varepsilon\right\} & =\sum_{j=0}^{\infty} P\left\{\|N-h\|_{2} \leq \varepsilon \mid N_{1}=j\right\} \frac{e^{-1}}{j !} \\
& =2^{k} e^{-1} \frac{\varepsilon^{2 k}}{k !}+O\left(\varepsilon^{2 k+2}\right),
\end{aligned}
$$

which completes the proof of the theorem.

\section{The case of a diffusion Poisson process}

Theorem 4.1. Given a standard Poisson process $N=\left\{N_{s} ; s \in[0,1]\right\}$, and given a jump function $h_{t}=\sum_{i=1}^{k} I_{\left[S_{i}, 1\right)}(t), t \in[0,1], 0<S_{1}<\ldots<$ $S_{k}<1$, consider the diffusion process

$$
X_{t}=\int_{0}^{t} X_{s} d s+N_{t}, \quad t \in[0,1]
$$

and the jump curve

$$
\phi_{t}^{h}=\int_{0}^{t} \phi_{s}^{h} d s+h_{t}, \quad t \in[0,1]
$$

Then, for $\varepsilon>0$ small enough,

$$
\begin{aligned}
& P\left\{\left\|X-\phi^{h}\right\|_{1} \leq \varepsilon\right\}=2^{k} e^{-1} \frac{\varepsilon^{k}}{k !}+O\left(\varepsilon^{k+1}\right), \\
& P\left\{\left\|X-\phi^{h}\right\|_{2} \leq \varepsilon\right\}=2^{k} e^{-1} \frac{\varepsilon^{2 k}}{k !}+O\left(\varepsilon^{2 k+2}\right) .
\end{aligned}
$$

The solution of such equations can be expressed, using the jump points of the Poisson process and the jump function, as

$$
X(t)=\sum_{i=1}^{\infty} e^{t-T_{i}} I_{\left[T_{i}, 1\right)}(t) \quad \text { and } \quad \phi_{t}^{h}=\sum_{i=1}^{k} e^{t-S_{i}} I_{\left[S_{i}, 1\right)}(t) .
$$

As in the case of Poisson process, we can assume that there exists $\varepsilon_{0}>0$ such that $S_{i+1}-S_{i}>\varepsilon_{0}^{2}$ for all $i \in\{1, \ldots, k-1\}, S_{1}>\varepsilon_{0}^{2}$ and $1-S_{k}>\varepsilon_{0}^{2}$. From now on we will assume $\varepsilon \leq \varepsilon_{0}$.

Before the proof of the theorem we will show a preliminary lemma. 
Lemma 4.2. (a) For fixed $\alpha<\varepsilon_{0}$, if there exists $i \in\{1, \ldots, k\}$ such that $\left|S_{i}-T_{i}\right|>\alpha^{2}$, then

$$
\left\|X-\phi^{h}\right\|_{2}^{2}>C_{1} \alpha^{2}
$$

where $C_{1}$ denotes a universal constant.

(b) There exists $\varepsilon_{1}>0$, depending only on $k$, such that for fixed $\alpha<\varepsilon_{1}$, if for some $i>k, T_{i}<1$ and $1-T_{i}>\alpha^{2}$, then

$$
\left\|X-\phi^{h}\right\|_{2}^{2}>C_{2} \alpha^{2}
$$

where $C_{2}$ is another universal constant.

Proof. (a) Set $n:=\inf \left\{i \in\{1, \ldots, k\}:\left|S_{i}-T_{i}\right|>\alpha^{2}\right\}$. Then

$$
\left\|X-\phi^{h}\right\|_{2}^{2} \geq \int_{S_{n}-\alpha^{2}}^{S_{n}+\alpha^{2}}\left|X(t)-\phi^{h}(t)\right|^{2} d t
$$

If we define

$$
d_{n}=\sum_{i=1}^{n-1}\left(e^{-T_{i}}-e^{-S_{i}}\right)+I_{\left\{T_{n}<S_{n}\right\}} e^{-T_{n}},
$$

the last integral is equal to

$$
\begin{array}{rl}
\int_{S_{n}-\alpha^{2}}^{S_{n}} e^{2 t} d_{n}^{2} & d t+\int_{S_{n}}^{S_{n}+\alpha^{2}} e^{2 t}\left(d_{n}-e^{-S_{n}}\right)^{2} d t \\
& =\frac{d_{n}^{2}}{2}\left(e^{2 S_{n}}-e^{2\left(S_{n}-\alpha^{2}\right)}\right)+\frac{\left(d_{n}-e^{-S_{n}}\right)^{2}}{2}\left(e^{2 S_{n}+\alpha^{2}}-e^{2 S_{n}}\right) \\
& \geq\left[\frac{d_{n}^{2}}{2}+\frac{\left(d_{n}-e^{-S_{n}}\right)^{2}}{2}\right] 2 \alpha^{2} \geq \frac{e^{-2 S_{n}}}{2} \alpha^{2}>\frac{e^{-2}}{2} \alpha^{2} ;
\end{array}
$$

we have used the fact that $e^{x}-e^{y} \geq x-y$ for $0<y<x$, and $a^{2}+b^{2} \geq$ $(a+b)^{2} / 2$ for all $a, b \in \mathbb{R}$.

(b) We can assume that $\left|S_{i}-T_{i}\right| \leq \alpha^{2}$ for all $i \in\{1, \ldots, k\}$. Notice that $d_{\infty}(t)=\left|\sum_{i=1}^{k}\left(e^{-T_{i}}-e^{-S_{i}}\right)+\sum_{i=k+1}^{\infty} e^{-T_{i}} I_{\left[T_{i}, 1\right)}(t)\right| \geq e^{-T_{k+1}}-k \alpha^{2} \geq e^{-1}-k \alpha^{2}$.

Then

$$
\begin{aligned}
\left\|X-\phi^{h}\right\|_{2}^{2} & >\int_{1-\alpha^{2}}^{1}|X(t)-\phi(t)|^{2} d t=\int_{1-\alpha^{2}}^{1} e^{2 t}\left|d_{\infty}(t)\right|^{2} d t \\
& \geq \frac{\left(e^{-1}-k \alpha^{2}\right)^{2}}{2}\left(e^{2}-e^{2\left(1-\alpha^{2}\right)}\right) \geq C_{2} \alpha^{2},
\end{aligned}
$$

by similar arguments to the proof of (a) and for $\alpha$ small enough. 
REMARK 4.3. Note that from Lemma 4.2 we can assume, for $\varepsilon$ small enough, that for all $i, l \in\{1, \ldots, k\}$ such that $i<l, S_{i}<T_{l}$ and $T_{i}<S_{l}$.

Proof of Theorem 4.1. As in the case of a Poisson process we only develop the $L^{2}$ case. We have

$$
P\left\{\left\|X-\phi^{h}\right\|_{2} \leq \varepsilon\right\}=\sum_{j=0}^{\infty} P\left\{\left\|X-\phi^{h}\right\|_{2} \leq \varepsilon \mid N_{1}=j\right\} \frac{e^{-1}}{j !} .
$$

To compute the probabilities involved in the sum we will consider three cases:

- If $j<k$, then for $\varepsilon$ small enough, by Lemma 4.2 ,

$$
P\left\{\left\|X-\phi^{h}\right\|_{2} \leq \varepsilon \mid N_{1}=j\right\}=0 .
$$

- If $j=k$, we will prove that $P\left\{\left\|X-\phi^{h}\right\|_{2} \leq \varepsilon \mid N_{1}=k\right\}=\left(2 \varepsilon^{2}\right)^{k}+$ $O\left(\varepsilon^{2 k+2}\right)$.

Notice first that by Lemma 4.2 we only need to consider the $\omega$ such that $\left|S_{i}-T_{i}(\omega)\right|<\varepsilon^{2} / C_{1}$ for all $i \in\{1, \ldots, k\}$.

Using the expressions for $X$ and $\phi^{h}$ involving the jump points, we have, for $N_{1}=k$,

$$
\begin{aligned}
\left\|X-\phi^{h}\right\|_{2}^{2}= & \int_{0}^{1} e^{2 t}\left(\sum_{i, l=1}^{k}\left(e^{-T_{i}} I_{\left[T_{i}, 1\right)}(t)-e^{-S_{i}} I_{\left[S_{i}, 1\right)}(t)\right)\right. \\
& \left.\times\left(e^{-T_{l}} I_{\left[T_{l}, 1\right)}(t)-e^{-S_{l}} I_{\left[S_{l}, 1\right)}(t)\right)\right) d t \\
= & \frac{1}{2}\left[\sum _ { i , l = 1 } ^ { k } \left\{e^{-T_{i}-T_{l}}\left(e^{2}-e^{2\left(T_{i} \vee T_{l}\right)}\right)-e^{-T_{i}-S_{l}}\left(e^{2}-e^{2\left(T_{i} \vee S_{l}\right)}\right)\right.\right. \\
& \left.\left.-e^{-S_{i}-T_{l}}\left(e^{2}-e^{2\left(S_{i} \vee T_{l}\right)}\right)+e^{-S_{i}-S_{l}}\left(e^{2}-e^{2\left(S_{i} \vee S_{l}\right)}\right)\right\}\right] \\
= & \frac{1}{2}\left[\sum _ { i , l = 1 } ^ { k } \left\{e^{2-T_{i}-T_{l}}-e^{\left|T_{i}-T_{l}\right|}-2 e^{2-T_{i}-S_{l}}+2 e^{\left|T_{i}-S_{l}\right|}\right.\right. \\
& \left.\left.+e^{2-S_{i}-S_{l}}-e^{\left|S_{i}-S_{l}\right|}\right\}\right] .
\end{aligned}
$$

Notice that if we put $\delta_{i}:=T_{i}-S_{i}$, then

$$
\begin{aligned}
e^{2-S_{i}-S_{l}}+e^{2-T_{i}-T_{l}}- & 2 e^{2-T_{i}-S_{l}}=e^{2-S_{i}-S_{l}}\left(1+e^{-\delta_{i}-\delta_{l}}-2 e^{-\delta_{i}}\right) \\
& =e^{2-\left(S_{i}+S_{l}\right)}\left(\delta_{i}-\delta_{l}+\frac{\left(\delta_{i}+\delta_{l}\right)^{2}}{2} e^{\eta_{i, l}^{1}}-\delta_{i}^{2} e^{\eta_{i}^{1}}\right),
\end{aligned}
$$

by Taylor's decomposition, where $\eta_{i}^{1}, \eta_{i, l}^{1} \in\left[-2 \varepsilon^{2} / C_{1}, 2 \varepsilon^{2} / C_{1}\right]$.

On the other hand, by Remark 4.3, for the other term involved in the sum (2) we have three situations: 
If $i<l$, then

$$
\begin{aligned}
2 e^{\left|T_{i}-S_{l}\right|}-e^{\left|T_{i}-T_{l}\right|}-e^{\left|S_{i}-S_{l}\right|} & =e^{S_{l}-S_{i}}\left(-e^{\delta_{l}-\delta_{i}}+2 e^{-\delta_{i}}-1\right) \\
& =e^{S_{l}-S_{i}}\left(-\delta_{l}-\delta_{i}-\frac{\left(\delta_{l}-\delta_{i}\right)^{2}}{2} e^{\eta_{i, l}^{2}}+\delta_{i}^{2} e^{\eta_{i}^{2}}\right),
\end{aligned}
$$

where $\eta_{i}^{2}, \eta_{i, l}^{2} \in\left[-2 \varepsilon^{2} / C_{1}, 2 \varepsilon^{2} / C_{1}\right]$.

If $i=l$, then

$$
2 e^{\left|T_{i}-S_{l}\right|}-e^{\left|T_{i}-T_{l}\right|}-e^{\left|S_{i}-S_{l}\right|}=2 e^{\left|T_{i}-S_{i}\right|}-2=2\left|\delta_{i}\right|+\delta_{i}^{2} e^{\eta_{i}^{3}},
$$

where $\eta_{i}^{3} \in\left[-2 \varepsilon^{2} / C_{1}, 2 \varepsilon^{2} / C_{1}\right]$.

Finally, if $i>l$, then

$$
\begin{aligned}
2 e^{\left|T_{i}-S_{l}\right|}-e^{\left|T_{i}-T_{l}\right|}-e^{\left|S_{i}-S_{l}\right|} & =e^{S_{i}-S_{l}}\left(-e^{\delta_{i}-\delta_{l}}+2 e^{\delta_{i}}-1\right) \\
& =e^{S_{i}-S_{l}}\left(\delta_{i}+\delta_{l}-\frac{\left(\delta_{i}-\delta_{l}\right)^{2}}{2} e^{\eta_{i, l}^{4}}+\delta_{i}^{2} e^{\eta_{i}^{4}}\right) .
\end{aligned}
$$

where $\eta_{i}^{4}, \eta_{i, l}^{4} \in\left[-2 \varepsilon^{2} / C_{1}, 2 \varepsilon^{2} / C_{1}\right]$.

So, when $N_{1}=k$,

$$
\left\|X-\phi^{h}\right\|_{2}^{2}=\sum_{i=1}^{k}\left|\delta_{i}\right|+Z
$$

where

$$
\begin{aligned}
Z= & \sum_{i, l=1}^{k} e^{-2\left(S_{i}+S_{l}\right)}\left(\frac{\left(\delta_{i}+\delta_{l}\right)^{2}}{2} e^{\eta_{i, l}^{1}}-\delta_{i}^{2} e^{\eta_{i}^{1}}\right) \\
& +\sum_{i=1}^{k} \delta_{i}^{2} e^{\eta_{i}^{3}}+\sum_{i<l} e^{S_{l}-S_{i}}\left(-\frac{\left(\delta_{l}-\delta_{i}\right)^{2}}{2} e^{\eta_{i, l}^{2}}+\delta_{i}^{2} e^{\eta_{i}^{2}}\right) \\
& +\sum_{i>l} e^{S_{i}-S_{l}}\left(-\frac{\left(\delta_{i}-\delta_{l}\right)^{2}}{2} e^{\eta_{i, l}^{4}}+\delta_{i}^{2} e^{\eta_{i}^{4}}\right) .
\end{aligned}
$$

For fixed $\omega$, by Lemma $4.2\left(\right.$ a), if $\left\|X(\omega)-\phi^{h}\right\|_{2} \leq \varepsilon$ we get $\left|T_{i}(\omega)-S_{i}\right| \leq$ $\varepsilon^{2} / C_{1}$ for all $i \in\{1, \ldots, k\}$. Then $|Z(\omega)|<C_{3} k^{2} \varepsilon^{4} / C_{1}^{2}$ with $C_{3}:=10 e^{1 / C_{1}}$, and using the results proved in Theorem 3.1 we get from (3)

$$
\begin{aligned}
P\left\{\left\|X-\phi^{h}\right\|_{2} \leq \varepsilon \mid N_{1}\right. & =k\} \leq P\left\{\sum_{i=1}^{k}\left|\delta_{i}\right| \leq \varepsilon^{2}+C_{3} k^{2} \frac{\varepsilon^{4}}{C_{1}^{2}} \mid N_{1}=k\right\} \\
& =\left(2 \varepsilon^{2}\left(1+C_{3} k^{2} \frac{\varepsilon^{2}}{C_{1}^{2}}\right)\right)^{k} \leq 2^{k} \varepsilon^{2 k}+c_{k, 1} \varepsilon^{2 k+2}
\end{aligned}
$$

where $c_{k, 1}$ depends only on $k$ and $C_{1}$. On the other hand, for fixed $\omega$ such that $\sum_{i=1}^{k}\left|\delta_{i}\right| \leq \varepsilon^{2}$ we clearly have $|Z(\omega)|<C_{3} k^{2} \varepsilon^{4}$. So, again from (3) we 
get

$$
\begin{aligned}
P\left\{\left\|X-\phi^{h}\right\|_{2} \leq \varepsilon \mid N_{1}=k\right\} & \geq P\left\{\sum_{i=1}^{k}\left|\delta_{i}\right| \leq \varepsilon^{2}-C_{3} k^{2} \varepsilon^{4} \mid N_{1}=k\right\} \\
& =\left(2 \varepsilon^{2}\left(1-C_{3} k^{2} \varepsilon^{2}\right)\right)^{k} \geq 2^{k} \varepsilon^{2 k}+c_{k, 2} \varepsilon^{2 k+2}
\end{aligned}
$$

where $c_{k, 2}$ depends only on $k$ and $C_{1}$. Putting (4) and (5) together we obtain

$$
P\left\{\left\|X-\phi^{h}\right\|_{2} \leq \varepsilon \mid N_{1}=k\right\}=\left(2 \varepsilon^{2}\right)^{k}+O\left(\varepsilon^{2 k+2}\right) .
$$

- To deal with the case $j>k$ notice that, again by Lemma 4.2, if $\left\|X(\omega)-\phi^{h}\right\|_{2} \leq \varepsilon$ then $\left|T_{i}(\omega)-S_{i}\right| \leq \varepsilon^{2} / C_{1}$ for all $i \in\{1, \ldots, k\}$ and $\left|1-T_{i}(\omega)\right| \leq \varepsilon^{2} / C_{2}$ for all $i \in\{k+1, \ldots, j\}$. So, using Proposition 2.2 we have

$$
\begin{aligned}
& P\left\{\left\|X-\phi^{h}\right\|_{2} \leq \varepsilon \mid N_{1}=j\right\} \\
& \quad \leq P\left\{\left\{\max _{1 \leq i \leq k}\left|T_{i}-S_{i}\right| \leq \varepsilon^{2} / C_{1}\right\} \cap\left\{\max _{k+1 \leq i \leq j}\left|1-T_{i}\right| \leq \varepsilon^{2} / C_{2}\right\} \mid N_{1}=j\right\} \\
& \quad \leq \frac{j ! 2^{k} \varepsilon^{2 j}}{C_{1}^{k} C_{2}^{j-k}},
\end{aligned}
$$

and then

$$
\begin{aligned}
\sum_{j=k+1}^{\infty} P\left\{\left\|X-\phi^{h}\right\|_{2}\right. & \left.\leq \varepsilon \mid N_{1}=j\right\} \frac{e^{-1}}{j !} \\
& \leq \sum_{j=k+1}^{\infty} \frac{e^{-1} 2^{k} \varepsilon^{2 j}}{C_{1}^{k} C_{2}^{j-k}}=e^{-1} 2^{k} \frac{1}{C_{2} C_{1}^{k}} \cdot \frac{\varepsilon^{2 k+2}}{1-\varepsilon^{2} C_{2}} .
\end{aligned}
$$

Putting (1), (6) and (7) together we finish the proof of the theorem.

\section{References}

X. Bardina, C. Rovira and S. Tindel (2000), Onsager-Machlup functional for stochastic evolution equations, preprint.

X. Bardina, C. Rovira and S. Tindel (2001), Onsager-Machlup functional for stochastic evolution equations in a class of norms, Ann. Inst. H. Poincaré, to appear.

R. N. Bhattacharya and E. C. Waymire (1990), Stochastic Processes with Applications, Wiley.

M. Capitaine (2000), On the Onsager-Machlup functional for elliptic diffusion processes, in: Séminaire de Probabilités 34, Lecture Notes in Math. 1729, Springer, 313-328.

N. Ikeda and S. Watanabe (1981), Stochastic Differential Equations and Diffusion Processes, North-Holland.

T. Lyons and O. Zeitouni (1999), Conditional exponential moments for iterated Wiener integrals, Ann. Probab. 27, 1738-1749.

E. Mayer-Wolf and O. Zeitouni (1993), Onsager Machlup functionals for non-trace-class SPDEs, Probab. Theory Related Fields 95, 199-216. 
L. Shepp and O. Zeitouni (1992), A note on conditional exponential moments and OnsagerMachlup functionals, Ann. Probab. 20, 652-654.

Departament de Matemàtiques

Universitat Autònoma de Barcelona

08193 Bellaterra, Barcelona, Spain

E-mail: bardina@mat.uab.es

Institut Galilée

Université Paris 13

Avenue J. B. Clément

93430 Villetaneuse, France

E-mail: tindel@math.univ-paris13.fr
Facultat de Matemàtiques

Universitat de Barcelona

Gran Via 585

08007 Barcelona, Spain

E-mail: rovira@cerber.mat.ub.es

Received on 18.10.2001 